\title{
Role of 2-month sputum smears in predicting culture conversion in pulmonary tuberculosis
}

\author{
W-J. Su**\#, J-Y. Feng**\#, Y-C. Chiu*, S-F. Huang* and Y-C. Lee*,\#
}

ABSTRACT: Sputum smears and culture conversion are frequently used to evaluate treatment response in pulmonary tuberculosis patients. Limited data are available on the evaluation of the correlation between under-treatment sputum smear results and culture conversion.

This prospective study included sputum culture-proven pulmonary tuberculosis patients at six hospitals in Taiwan. At least two sets of sputum were collected at the completion of 8 weeks of TB treatment. The sensitivities and specificities of 2-month sputum smears were estimated based on culture conversion status.

A total of $\mathbf{3 7 1}$ patients were enrolled for analysis. Factors associated with culture conversion included having a smear positive before treatment, presence of a cavity on radiography, rifampicin resistance and usage of the DOTS (directly observed therapy, short course) strategy. The sensitivities of 2-month sputum smears for culture conversion among all patients, initially smear-positive patients and initially smear-negative patients were $64.3,71.4$ and $38 \%$, respectively, and the specificities were $81.6,69.9$ and $92.8 \%$, respectively. In patients who were 2-month sputum smear-positive, the 2-month culture conversion rate was $80 \%$ if the patients were under DOTS and without cavitary lesions in radiograms.

The predictive value of 2-month sputum smears in culture conversion was limited and highly influenced by clinical factors in pulmonary tuberculosis patients.

KEYWORDS: Culture, directly observed therapy short course implementation, Mycobacterium tuberculosis, pulmonary tuberculosis, sputum

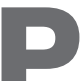
ulmonary tuberculosis (PTB) is an airborne infectious disease that requires multiple-drug combination therapy and long treatment duration. Despite great advances in molecular diagnosis, sputum cultures of $\mathrm{Myco}^{-}$ bacterium tuberculosis (MTB) remain the gold standard for PTB diagnosis. After initiation of anti-tuberculosis (anti-TB) treatment, sputum acidfast bacilli (AFB) smears and cultures of MTB are regularly checked during the treatment period and serve as important indicators of treatment response.

Achievement of sputum sterilisation, which is determined by sputum culture conversion, is a cardinal index of treatment success. Previous studies have demonstrated that the time to sputum negativity is an important determinant of relapse [1-3]. Failure to achieve sputum culture conversion in $<2$ months will lead to a worse treatment outcome [4]. Documentation of sputum culture conversion is also recommended as a necessary criterion before completion of anti-TB treatment in current treatment guidelines [5]. Based on the high predictive value of sputum culture conversion in treatment response $[4,6,7]$, identification of the clinical factors that are associated with a lower sputum culture conversion rate is of crucial importance in PTB management [8-10].

In spite of the high accuracy of sputum culture conversion in the assessment of treatment response, the long yield time limits its usefulness in clinical practice. As a simple and rapid test, sputum smears are frequently adopted as a substitute for sputum cultures to evaluate infectivity and treatment effectiveness. However, positive sputum AFB smears with negative results for MTB culture are not unusual during the course of anti-TB treatment. A false-positive sputum AFB smear (AFB smear positive but MTB culture negative) may lead to the physician making the wrong judgement and thereby wasting medical resources unnecessarily. Therefore, the predictive value of sputum AFB smears in

\section{AFFILIATIONS}

*Dept of Chest Medicine, Taipel Veterans General Hospital, and \#School of Medicine, National YangMing University, Taipei, Taiwan.

CORRESPONDENCE

W-J. Su

Dept of Chest Medicine

Taipei Veterans General Hospital

No. 201

Sec. 2

Shih-Pai Rd

Taipei 112

Taiwan

E-mail: wjsu@vghtpe.gov.tw

Received:

Jan 152010

Accepted after revision:

May 052010

First published online:

June 012010 
culture conversion and MTB infectivity under anti-TB treatment needs to be further clarified.

The purpose of this study was to elucidate the value of 2month sputum AFB smear status in predicting 2-month culture conversion under anti-TB treatment. We also investigated the clinical characteristics that were associated with 2-month culture conversion. The factors involved in discordant AFB smears and culture results were also explored.

\section{MATERIALS AND METHODS \\ Patients and setting}

This prospective, observational study was conducted in six hospitals in Taiwan, including five referral medical centres and one regional hospital that specialises in pulmonary diseases. Sputum culture-proven PTB patients from January 2007 to December 2008 were eligible for this study. The demographic profiles (age, sex and comorbidities) and clinical characteristics (presenting symptoms and signs, history of previous anti-TB treatment, and smoking habits) were obtained from medical records or telephone contact. The chest radiographs were reviewed by the responsible physician at each hospital. The adherence to directly observed therapy, short course (DOTS) strategy of each patient was determined according to the records in the Centers for Disease Control (CDC) registration database, Taiwan. Only patients who joined the DOTS programme immediately after CDC registration and without interruption throughout the treatment course for the initial 2 months were defined as adhering to the DOTS strategy. The institutional review boards of all six hospitals approved the study and informed consent was obtained from each patient before enrolment.

\section{Treatment of PTB}

All of the patients were treated with standard anti-TB treatment that included isoniazid, rifampicin, ethambutol and pyrazinamide in the initial phase. The dosage of the anti-TB drugs was adjusted according to the presence of concomitant renal disease and the regimen was modified when drug susceptibility results were available or when significant adverse effects occurred. At least two sets of sputum were collected for AFB smears and MTB cultures at the end of the eighth week after initiation of anti-TB treatment. Further sputum examinations after 2 months, including sputum smears and cultures, were ordered according to the decision made by the responsible physicians. The patients enrolled in this study were prospectively followed up until they had completed treatment. 2-month sputum culture conversion was defined as all collected sputum at 2 months being culturenegative for MTB, and there being no positive cultures thereafter until completion of the anti-TB treatment [11]. The sensitivity and specificity of the 2-month sputum AFB smears were calculated according to the results of the sputum cultures.

\section{Mycobacteriology}

Sputum AFB smears and cultures were performed in each hospital using standard methods. Sputum samples were decontaminated and liquefied with $3 \% \mathrm{NaOH}$, neutralised with $\mathrm{HCl}$ and then concentrated by centrifugation for $15 \mathrm{~min}$ at $3,000 \times g$. Sediments were resuspended in phosphate buffer, and smears and cultures were made from the resuspended material. Sputum smears were examined by Ziehl-Neelsen staining. The isolation of MTB in sputum cultures was performed in liquid medium (BACTEC; BD Diagnostic Systems, Sparks, MD, USA) and/or Lowenstein-Jensen (LJ) solid medium. Drug susceptibility testing was performed by the proportion method with Middlebrook $7 \mathrm{H} 11$ media. All clinical isolates were genotyped by commercial spoligotyping kit (Isogen Bioscience B.V., Maarssen, the Netherlands). The "Beijing strain" was defined as deletion from spacer 1 to spacer 34 in the direct repeat region and the presence of (at least three of) spacers 35-43.

\section{Data analysis}

Comparisons of demographic and clinical characteristics were done using a Chi-squared test or Fisher's exact test for categorical variables and the two-tailed independent (unpaired) t-test for continuous variables. Binary logistic regression analysis was performed to determine the independent variables and odds ratios with their $95 \%$ confidence intervals were presented. Significance was defined as $p<0.05$ (two-tailed). Statistical analysis was performed using a statistical software package (SPSS version 14.0; SPSS Inc., Chicago, IL, USA).

\section{RESULTS}

From January 2007 to December 2008, 524 sputum culturepositive PTB patients were enrolled from six hospitals. During the follow-up period, 49 patients were excluded because of mortality $<2$ months after initiation of anti-TB treatment and 104 patients were excluded due to incomplete smear and culture data at 2 months. Finally, 371 patients who met the selection criteria with available 2-month smear and culture results were enrolled for analysis (fig. 1). Of these patients, 279 had negative sputum MTB cultures at the end of the second month, but six of them had sputum reversion thereafter. Therefore, 273 patients met the criteria of 2-month sputum culture conversion and the overall 2-month conversion rate was $73.6 \%$.

The demographic characteristics of patients with or without 2 month sputum culture conversion are shown in table 1. Compared with the patients with 2-month sputum culture conversion, those without conversion were more likely to be male $(p=0.001)$, have a previous anti-TB history $(p=0.014)$, have a smoking habit $(\mathrm{p}=0.003)$, be sputum smear-positive before treatment $(p<0.001)$, presence of cavity on chest radiographs $(p<0.001)$, have body weight loss $(p=0.03)$, have rifampicin resistance $(\mathrm{p}<0.001)$, have multidrug-resistant tuberculosis (MDR-TB) isolates $(p=0.007)$ and were less likely to be under DOTS strategy $(p=0.001)$. The underlying comorbidities were comparable between patients with or without 2-month culture conversion. The proportion of Beijing strain infection was also similar between the two subgroups. There were no statistical differences in demographic data between the included and excluded patients (see Appendix).

The disagreement of 2-month sputum AFB smears and culture conversion is shown in table 2. In 113 patients who were 2-month sputum smear-positive, 63 patients had positive sputum cultures with growth of MTB at or after 2 months, seven had positive sputum cultures with growth of nontuberculosis mycobacterium (NTM), and 43 had negative 


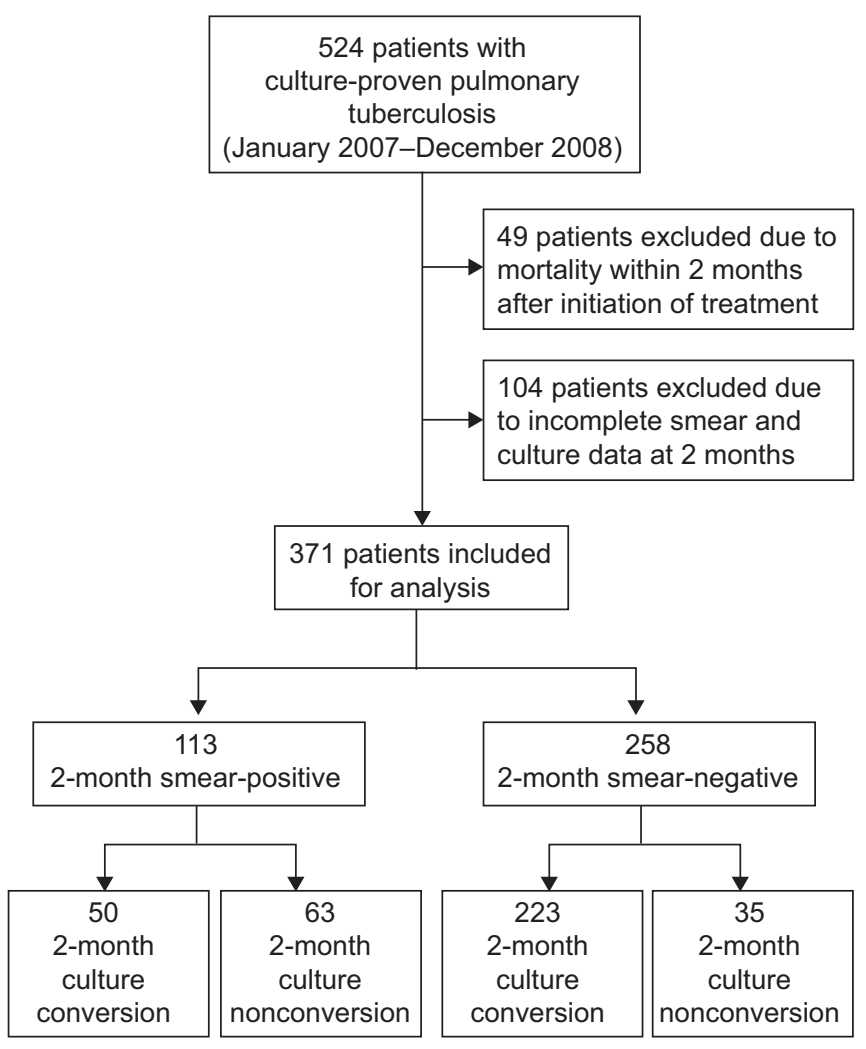

FIGURE 1. Study profile demonstrating the number of cases and reasons for exclusion.

sputum MTB cultures until completion of anti-TB treatment. Therefore, $50(44.2 \%)$ patients were proven to be culture converted, even though the 2-month AFB smears were positive. Meanwhile, in 258 patients who were 2-month sputum smear-negative, $223(86.4 \%)$ achieved 2-month culture conversion. The sensitivity and specificity of 2-month sputum smears in predicting 2-month culture conversion were $64.3 \%$ and $81.6 \%$, respectively. We further stratified the patients into subgroups according to the results of pre-treatment sputum AFB smears. In patients who were pre-treatment sputum smear-negative, the sensitivity and specificity of 2-month sputum smears were $38 \%$ and $92.8 \%$, respectively. In those who were pre-treatment sputum smear-positive, the sensitivity and specificity of 2-month sputum smears were $71.4 \%$ and $69.9 \%$, respectively.

After logistical regression analysis, the independent risk factors associated with failure of 2-month sputum culture conversion included initial sputum AFB smear positivity (OR $0.28,95 \%$ CI $0.15-0.52$ ), presence of cavity on radiographs (OR $0.35,95 \%$ CI $0.20-0.63$ ) and rifampicin resistance (OR $0.31,95 \%$ CI 0.14-0.67) (table 3). In contrast, patients under DOTS strategy were more likely to achieve 2 -month sputum culture conversion (OR 3.57, 95\% CI 1.94-6.55; p<0.001). In patients who were 2-month sputum smear-positive, those without cavitary lesions (OR $0.24,95 \%$ CI $0.10-0.59$ ), with lobar/ segmental consolidation (OR 3.28, 95\% CI 1.02-10.48) and under DOTS strategy (OR 4.41, 95\% CI 1.62-12.02) were more likely to achieve culture conversion.
The 2-month culture conversion rates among various clinical settings are shown in table 4 . In patients who were 2-month sputum smear-positive, the culture conversion rate was as high as $80 \%$ in those without cavitary lesions combined with usage of DOTS strategy, and as low as $18.2 \%$ in those without DOTS strategy combined with cavitary lesions. In patients who were 2-month sputum smear-negative, the culture conversion rate ranged from 71.8 to $91.3 \%$, and those without cavitary lesions in chest radiographs combined with negative initial sputum smear had the highest culture conversion rate.

\section{DISCUSSION}

Regularly followed sputum smears and cultures during antiTB treatment are thought to be the major, and also the most reliable, measures to evaluate treatment effectiveness. The present study demonstrated that being initially sputum AFB smear-negative, without cavitary lesions in chest radiographs, susceptible to rifampicin and under DOTS strategy were independent factors associated with 2-month sputum culture conversion in PTB patients. Our study also demonstrated a remarkable discordance between 2-month sputum AFB smear results and 2-month culture conversion. Both the sensitivity and specificity of 2-month sputum smears were not high enough to predict 2-month culture conversion. Our results highlight the importance of clinical characteristics in determining 2-month culture conversion and 2-month sputum smear results should not be utilised alone as an indicator of treatment response.

Delayed sputum culture conversion indicates poor treatment response and represents the persistent possibility of MTB bacilli transmission from the patients to other people. Knowing the risk factors associated with delayed culture conversion helps us to identify the patients that require the most medical resources and attention, such as prolonged respiratory isolation. Several previous studies have evaluated the factors that lead to a delay in sputum culture conversion [11-14]. Although the definition of sputum conversion and schedules for sputum monitoring varied in each study, cavitary disease and numerous AFB on the initial smears were the most frequently identified factors. In line with these reports, our study found that positive results of initial sputum smears, presence of cavity in chest radiographs, rifampicin resistance, MDR-TB isolates and treatment without DOTS strategy were independent risk factors of failed 2-month sputum culture conversion. Most of these factors can be identified in the early stage of antiTB treatment and provide clinicians with an opportunity to take more aggressive action to treat these patients, such as DOTS strategy or even surgery [15]. Our findings, combined with previous reports, suggest that these risk factors have remained similar globally for decades, despite the advances of tuberculosis management in recent years. It also reminds us that PTB patients with these characteristics are highly infectious and more effective treatment is eagerly needed.

In the present study, we also evaluated the impact of genotyping in 2-month sputum culture conversion. Among various genotypes of MTB, the Beijing strain has been most extensively studied around the world. A higher drugresistance rate of the Beijing strain has been reported in some areas, but the resistance rate and resistance patterns vary from 
TABLE 1 Demographic data of pulmonary tuberculosis (TB) patients with or without 2-month culture conversion

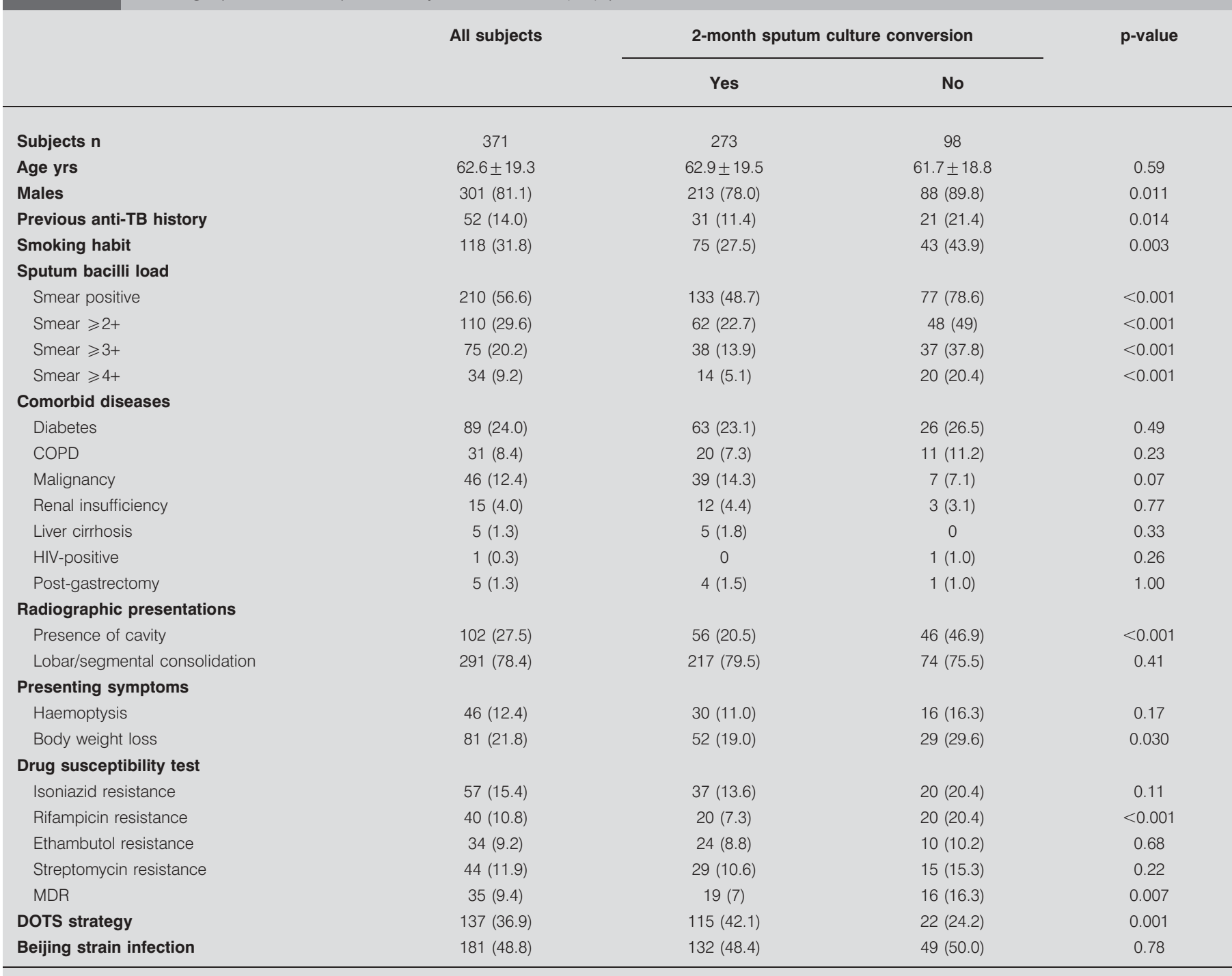

Data are presented as mean \pm SD or $n(\%)$, unless otherwise stated. COPD: chronic obstructive pulmonary disease; DOTS: directly observed therapy, short course; MDR: multidrug resistance.

area to area [16, 17]. Studies evaluating the clinical impact of the Beijing genotype have also reported inconsistent results [18-20]. Although studies from Vietnam and Taiwan have reported higher treatment failure and relapse rates among patients infected with the Beijing strain [21,22], the 2-month sputum culture conversion rates were comparable between patients infected with the Beijing and non-Beijing strains in the present study. According to the present data, the impact of the Beijing strain in 2-month sputum culture conversion is probably limited in Taiwan. However, previous studies have documented that the Beijing strain, in Taiwan, is not associated with a significantly higher resistance rate to rifampicin, which may lead to its nonsignificant role in culture conversion [21, 23]. In areas where the Beijing strain has been reported to have a higher drug-resistance rate, especially to rifampicin, the role of genotyping in culture conversion remains to be identified and further studies are needed to clarify the issue.
Sputum microscopy is fast, simple, inexpensive and has been widely adopted in MTB endemic areas. More importantly, sputum smear results represent the bacterial load and help to identify the most infectious patients. According to previous reports, the specificity of sputum AFB smears is very high, approaching $100 \%$ [24, 25]. In contrast, the sensitivity of sputum microscopy is lower and varies from 40 to $80 \%$, depending on the method used [26,27]. It should be noted that most of these studies were conducted in the setting of pretreatment case diagnosis. In our study, the sensitivity of 2 month sputum smears was $64.3 \%$, which is comparable to the previous reports. However, the specificity was only $81.6 \%$, which is much lower than the reports in the setting of pretreatment case diagnosis. When dividing the patients into subgroups according to their initial sputum smear results, the specificity declined further to $69.9 \%$ in those who were initially sputum smear-positive. Because only sputum culture positive 


\begin{tabular}{|c|c|c|c|c|c|c|}
\hline \multirow{2}{*}{ TABLE 2} & \multirow[t]{2}{*}{ Subjects } & \multirow[t]{2}{*}{ 2-month smear results } & \multicolumn{2}{|c|}{ 2-month culture conversion } & \multirow[t]{2}{*}{ Sensitivity \% } & \multirow[t]{2}{*}{ Specificity \% } \\
\hline & & & No & Yes & & \\
\hline Overall patients & 371 & & & & 64.3 & 81.6 \\
\hline \multirow[t]{3}{*}{ Pre-treatment smear positive } & 210 & & & & 71.4 & 69.9 \\
\hline & & Positive & 55 & 40 & & \\
\hline & & Negative & 22 & 93 & & \\
\hline \multirow[t]{2}{*}{ Pre-treatment smear negative } & 161 & & & & 38.0 & 92.8 \\
\hline & & Positive & 8 & 10 & & \\
\hline
\end{tabular}

Data are presented as n, unless otherwise stated

patients were included, the sensitivity and specificity of pretreatment sputum smears could not be calculated in the present study. However, our results indicated the limitation of sputum AFB smears when used to assess the treatment response and infectivity during the course of anti-TB treatment. We also demonstrated that the sensitivity and specificity of 2-month sputum AFB smears were heavily affected by the pre-treatment sputum smear results.

The persistent presence of AFB in sputum during PTB treatment is not a rare condition in clinical practice. Many factors contribute to the discordance between sputum AFB smears and cultures during the course of anti-TB treatment. The decline of bacilli in sputum after effective treatment decreases the sensitivity of 2-month sputum AFB smears. Nonviable bacilli in sputum and NTM colonisation may lower the specificity as well [28-30]. Visible AFB in sputum with negative results from sputum MTB culture occurred in 50 patients in our study, including 40 patients who were pretreatment sputum AFB smear-positive and 10 patients who were pre-treatment sputum AFB smear-negative. Among these 50 patients, growth of NTM occurred in seven patients and the remaining 43 patients, results were speculated to be due to nonviable bacilli in the sputum. The probabilities of inappropriate culture process or specimen contamination were low, as these patients were evenly distributed across the six study hospitals.

According to our data and previous reports, clinicians should be cautious in interpreting the results of sputum AFB smears after initiation of effective anti-TB treatment. When the sputum smears are positive but the culture results remain unavailable, what kind of information can help clinicians to assess treatment response? A previous report found that if the

TABLE 3 Predictors of 2-month culture conversion in patients who were 2-month sputum smear-positive or -negative

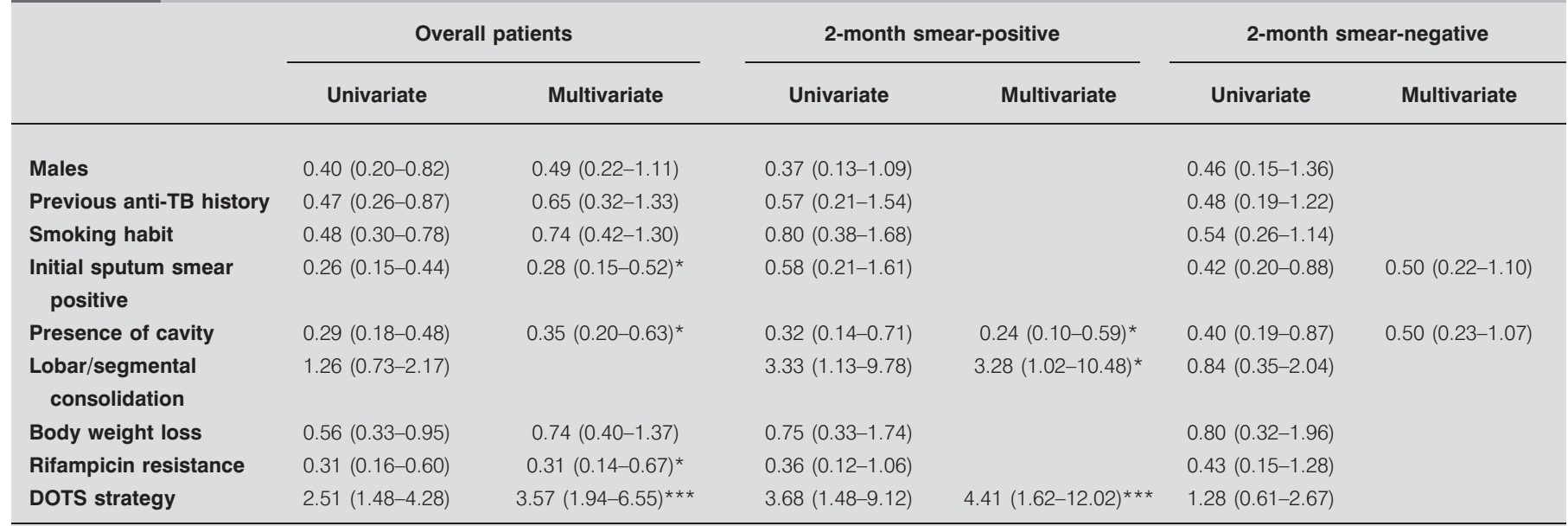

Data are presented as OR $(95 \% \mathrm{Cl})$. TB: tuberculosis; DOTS: directly observed therapy, short course. *: $\mathrm{p}<0.05$; ***: $\mathrm{p}<0.001$. 
TABLE 4 2-month culture conversion rates among various clinical settings

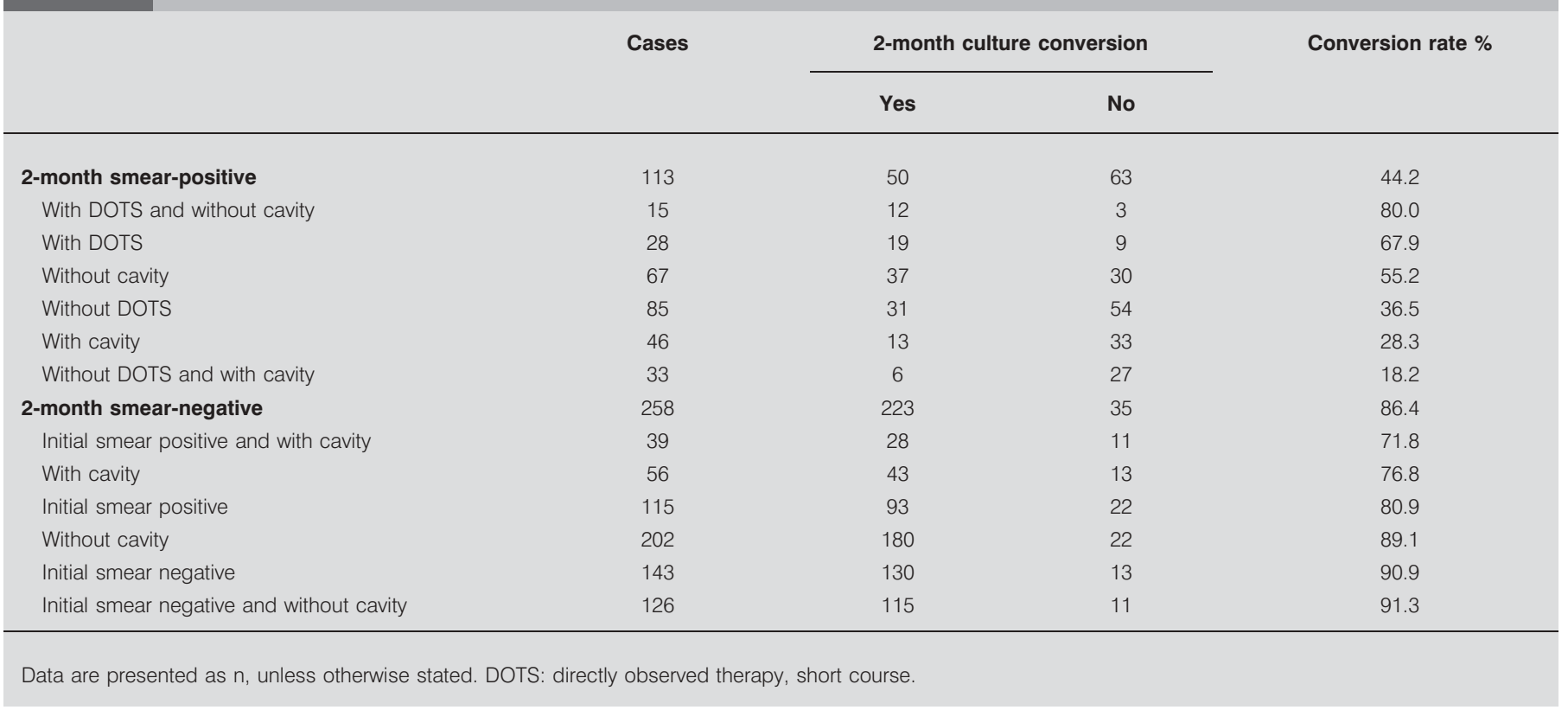

treatment regimen is effective, the patients are compliant to the medication, and the radiographs show improvement, then the positive sputum smears are usually associated with negative culture results [30]. The present study reported that the 2-month culture-conversion rate could be as high as $80 \%$ when the patients were under DOTS strategy and the initial chest plain film showed no cavitary lesions, even if the 2month sputum smear was positive. In contrast, we also showed that the impact of clinical characteristics were relatively low if the 2-month sputum AFB smears were negative. Our findings highlight the importance of clinical judgment in interpreting the positive results of sputum smears during the course of anti-TB treatment. Basing judgements on the results of sputum smears alone may lead to excess length of stay in isolation rooms. In addition, patients and clinicians may suffer from unnecessary anxiety and prolongation of treatment duration. A new measure with high accuracy and short yield time is also urgently required for treatment response and infectivity evaluation during anti-TB treatment.

Our study has several limitations. Considering the possible bias in chest radiogram interpretation, we did not evaluate the impact of disease extensiveness on culture conversion in this multisite study. We did not record the duration of presenting symptoms at diagnosis of PTB in patient enrolment. Meanwhile, the sputum MTB cultures were performed with liquid (BACTEC) and/or the solid (LJ) medium depending on the availability at each hospital. Compared with solid culture medium, liquid medium is reported to have a higher sensitivity but also higher NTM contamination rate $[31,32]$. The differences between liquid and solid culture medium may have lead to bias in the determination of culture conversion and detection of NTM in sputum specimens between hospitals.
In conclusion, our study found that the main independent determinants of 2-month sputum culture conversion included initial sputum smear results, cavity formation in radiographs, rifampicin resistance, MDR-TB isolates and usage of DOTS strategy. Intensified treatment and transmission precautions are especially required in patients with these characteristics. We also demonstrated that the ability of sputum AFB smears to predict culture conversion was limited during the course of anti-TB treatment. Physicians should take clinical characteristics into consideration when interpreting the results of post-treatment sputum smears.

\section{APPENDIX}

Demographic data obtained from the patients included and excluded in the study are provided in table 5 .

\section{SUPPORT STATEMENT}

This study was funded by the Institute for Biotechnology and Medicine Industry, and the Taipei Veterans General Hospital (both Taipei, Taiwan) (grants V97C1-064, V98C1-039, V98A-086 and V99C1-181).

\section{STATEMENT OF INTEREST}

None declared.

\section{ACKNOWLEDGEMENTS}

We gratefully acknowledge K-W. Yu (Division of Clinical Microbiology, Dept of Pathology and Laboratory Medicine, Taipei Veterans General Hospital, Taipei, Taiwan), R-M. Huang (Chest Hospital, Dept of Health, Tainan, Taiwan), C-H. Lin (Division of Chest Medicine, Dept of Internal Medicine, Changhua Christian Hospital, Changhua, Taiwan), J-J. Hwang (Division of Pulmonary and Critical Care, Dept of Internal Medicine, Kaohsiung Medical University, Kaohsiung, Taiwan), J-J. Lee (Dept of Laboratory Medicine, Buddhist Tzu Chi General Hospital, Hualien, Taiwan) and M-C. Yu 


\begin{tabular}{|c|c|c|c|c|}
\hline \multirow[t]{2}{*}{ TABLE 5} & \multicolumn{4}{|c|}{$\begin{array}{l}\text { Demographic data of the included and excluded } \\
\text { patients }\end{array}$} \\
\hline & & $\begin{array}{l}\text { Included } \\
\text { patients }\end{array}$ & $\begin{array}{l}\text { Excluded } \\
\text { patients }\end{array}$ & $\mathrm{p}$-value \\
\hline \multicolumn{2}{|l|}{ Subjects $n$} & 371 & 104 & \\
\hline \multicolumn{2}{|l|}{ Age yrs } & $62.6 \pm 19.3$ & $60.2 \pm 19.7$ & 0.27 \\
\hline \multicolumn{2}{|l|}{ Males } & $301(81.1)$ & 77 (74) & 0.11 \\
\hline \multicolumn{2}{|c|}{ Previous anti-TB history } & $52(14.0)$ & $11(10.6)$ & 0.36 \\
\hline \multicolumn{2}{|c|}{ Smoking habit } & $118(31.8)$ & $27(26)$ & 0.25 \\
\hline \multicolumn{5}{|c|}{ Sputum bacilli load } \\
\hline \multicolumn{2}{|c|}{ Smear positive } & $210(56.6)$ & $52(50)$ & 0.23 \\
\hline \multicolumn{2}{|c|}{ Smear $\geqslant 2+$} & $110(29.6)$ & $26(25)$ & 0.35 \\
\hline \multicolumn{2}{|c|}{ Smear $\geqslant 3+$} & 75 (20.2) & $19(18.3)$ & 0.66 \\
\hline \multicolumn{2}{|c|}{ Smear $\geqslant 4+$} & $34(9.2)$ & $9(8.7)$ & 0.87 \\
\hline \multicolumn{5}{|c|}{ Comorbid diseases } \\
\hline \multicolumn{2}{|c|}{ Diabetes } & $89(24.0)$ & $23(22.1)$ & 0.69 \\
\hline \multicolumn{2}{|l|}{ COPD } & $31(8.4)$ & $10(9.6)$ & 0.69 \\
\hline \multicolumn{2}{|c|}{ Malignancy } & $46(12.4)$ & $12(11.5)$ & 0.81 \\
\hline \multicolumn{2}{|c|}{ Renal insufficiency } & $15(4.0)$ & $4(3.8)$ & 0.93 \\
\hline \multicolumn{2}{|c|}{ Liver cirrhosis } & $5(1.3)$ & $1(1)$ & 0.76 \\
\hline \multicolumn{2}{|c|}{ HIV-positive } & $1(0.3)$ & 0 & 1.00 \\
\hline \multicolumn{2}{|c|}{ Post-gastrectomy } & $5(1.3)$ & $1(1)$ & 0.76 \\
\hline \multicolumn{5}{|c|}{ Radiographic presentations } \\
\hline \multicolumn{2}{|c|}{ Presence of cavity } & $102(27.5)$ & $23(22.1)$ & 0.27 \\
\hline \multicolumn{2}{|c|}{ Lobar/segmental consolidation } & $291(78.4)$ & $76(73.1)$ & 0.25 \\
\hline \multicolumn{5}{|c|}{ Presenting symptoms } \\
\hline \multicolumn{2}{|c|}{ Haemoptysis } & $46(12.4)$ & $15(14.4)$ & 0.59 \\
\hline \multicolumn{2}{|c|}{ Body weight loss } & $81(21.8)$ & $16(15.4)$ & 0.15 \\
\hline \multicolumn{5}{|c|}{ Drug susceptibility test } \\
\hline \multicolumn{2}{|c|}{ Isoniazid resistance } & $57(15.4)$ & $22(21.2)$ & 0.16 \\
\hline \multicolumn{2}{|c|}{ Rifampicin resistance } & $40(10.8)$ & $12(11.5)$ & 0.83 \\
\hline \multicolumn{2}{|c|}{ Ethambutol resistance } & $34(9.2)$ & $13(12.5)$ & 0.31 \\
\hline Streptomy & resistance & $44(11.9)$ & $15(14.4)$ & 0.48 \\
\hline MDR & & $35(9.4)$ & $12(11.5)$ & 0.53 \\
\hline DOTS strate & & $137(36.9)$ & $40(38.5)$ & 0.76 \\
\hline Beijing stra & infection & $181(48.8)$ & $51(49)$ & 0.96 \\
\hline
\end{tabular}

Data are presented as mean \pm SD or $n(\%)$, unless otherwise stated. TB: tuberculosis; COPD: chronic obstructive pulmonary disease; DOTS: directly observed therapy, short course; MDR: multidrug resistance.

(Dept of Medicine, Wan-Fang Hospital, Taipei, Taiwan) for their help in the study design and patient enrolment.

\section{REFERENCES}

1 Singla R, Osman MM, Khan N, et al. Factors predicting persistent sputum smear positivity among pulmonary tuberculosis patients 2 months after treatment. Int J Tuberc Lung Dis 2003; 7: 58-64.

2 Zierski M, Bek E, Long MW, et al. Short-course (6 month) cooperative tuberculosis study in Poland: results 18 months after completion of treatment. Am Rev Respir Dis 1980; 122: 879-889.

3 Cao JP, Zhang LY, Zhu JQ, et al. Two-year follow-up of directlyobserved intermittent regimens for smear-positive pulmonary tuberculosis in China. Int J Tuberc Lung Dis 1998; 2: 360-364.

4 Holtz TH, Sternberg M, Kammerer S, et al. Time to sputum culture conversion in multidrug-resistant tuberculosis: predictors and relationship to treatment outcome. Ann Intern Med 2006; 144: 650-659.
5 Taylor Z, Nolan CM, Blumberg HM, et al. Controlling tuberculosis in the United States. Recommendations from the American Thoracic Society, CDC, and the Infectious Diseases Society of America. MMWR Recomm Rep 2005; 54: 1-81.

6 Sevim T, Ataç G, Güngör G, et al. Treatment outcome of relapse and defaulter pulmonary tuberculosis patients. Int J Tuberc Lung Dis 2002; 6: 320-325.

7 Lienhardt C, Manneh K, Bouchier V, et al. Factors determining the outcome of treatment of adult smear-positive tuberculosis cases in The Gambia. Int J Tuberc Lung Dis 1998; 2: 712-718.

8 Güler M, Unsal E, Dursun B, et al. Factors influencing sputum smear and culture conversion time among patients with new case pulmonary tuberculosis. Int J Clin Pract 2007; 61: 231-235.

9 Fortún J, Martín-Dávila P, Molina A, et al. Sputum conversion among patients with pulmonary tuberculosis: are there implications for removal of respiratory isolation? J Antimicrob Chemother 2007; 59: 794-798.

10 Salihu HM, Aliyu MH, Ratard R, et al. Characteristics associated with reported sputum culture conversion in the era of re-emergent Mycobacterium tuberculosis in the State of North Carolina, 19931998. Int J Tuberc Lung Dis 2003; 7: 1070-1076.

11 Liu Z, Shilkret KL, Ellis HM. Predictors of sputum culture conversion among patients with tuberculosis in the era of tuberculosis resurgence. Arch Intern Med 1999; 159: 1110-1116.

12 Domínguez-Castellano A, Muniain MA, Rodriguez-Baño J, et al. Factors associated with time to sputum smear conversion in active pulmonary tuberculosis. Int J Tuberc Lung Dis 2003; 7: 432-438.

13 Telzak EE, Fazal BA, Pollard CL, et al. Factors influencing time to sputum conversion among patients with smear-positive pulmonary tuberculosis. Clin Infect Dis 1997; 25: 666-670.

14 Abal AT, Jayakrishnan B, Parwer S, et al. Effect of cigarette smoking on sputum smear conversion in adults with active pulmonary tuberculosis. Respir Med 2005; 99: 415-420.

15 Chan ED, Laurel V, Strand MJ, et al. Treatment and outcome analysis of 205 patients with multidrug-resistant tuberculosis. Am J Respir Crit Care Med 2004; 169: 1103-1109.

16 European Concerted Action on New Generation Genetic Markers and Techniques for the Epidemiology and Control of Tuberculosis. Beijing/W genotype Mycobacterium tuberculosis and drug resistance. Emerg Infect Dis 2006; 12: 736-743.

17 Glynn JR, Whiteley J, Bifani PJ, et al. Worldwide occurrence of Beijing/W strains of Mycobacterium tuberculosis: a systematic review. Emerg Infect Dis 2002; 8: 843-849.

18 Van Crevel R, Nelwan RH, de Lenne W, et al. Mycobacterium tuberculosis Beijing genotype strains associated with febrile response to treatment. Emerg Infect Dis 2001; 7: 880-883.

19 Sun YJ, Lim TK, Ong AK, et al. Tuberculosis associated with Mycobacterium tuberculosis Beijing and non-Beijing genotypes: a clinical and immunological comparison. BMC Infect Dis 2006; 6: 105.

20 Drobniewski F, Balabanova Y, Nikolayevsky V, et al. Drugresistant tuberculosis, clinical virulence, and the dominance of the Beijing strain family in Russia. JAMA 2005; 293: 2726-2731.

21 Feng JY, Su WJ, Tsai CC, et al. Clinical impact of Mycobacterium tuberculosis W-Beijing genotype strain infection on aged patients in Taiwan. J Clin Microbiol 2008; 46: 3127-3129.

22 Lan NTN, Lien HTK, Tung LB, et al. Mycobacterium tuberculosis Beijing genotype and risk for treatment failure and relapse, Vietnam. Emerg Infect Dis 2003; 9: 1633-1635.

23 Jou R, Chiang CY, Huang WL. Distribution of the Beijing family genotypes of Mycobacterium tuberculosis in Taiwan. J Clin Microbiol 2005; 43: 95-100.

24 Chakravorty S, Dudeja M, Hanif M, et al. Utility of universal sample processing methodology, combining smear microscopy, culture, and PCR, for diagnosis of pulmonary tuberculosis. J Clin Microbiol 2005; 43: 2703-2708. 
25 Fodor T. Detection of mycobacteria in sputum smears prepared by cytocentrifugation and sedimentation. Tuber Lung Dis 1995; 76: 273-274.

26 Bruchfeld J, Aderaye G, Palme IB, et al. Sputum concentration improves diagnosis of tuberculosis in a setting with a high prevalence of HIV. Trans $R$ Soc Trop Med Hyg 2000; 94: 677-680

27 Apers L, Mutsvangwa J, Magwenzi J, et al. A comparison of direct microscopy, the concentration method and the Mycobacteria Growth Indicator Tube for the examination of sputum for acidfast bacilli. Int J Tuberc Lung Dis 2003; 7: 376-381.

28 Vidal R, Martin-Casabona N, Juan A, et al. Incidence and significance of acid-fast bacilli in sputum smears at the end of antituberculous treatment. Chest 1996; 109: 1562-1565.
29 Lee JS, Kim EC, Joo SI, et al. The incidence and clinical implication of sputum with positive acid-fast bacilli smear but negative in mycobacterial culture in a tertiary referral hospital in South Korea. J Korean Med Sci 2008; 23: 767-771.

30 Al-Moamary MS, Black W, Bessuille E, et al. The significance of the persistent presence of acid-fast bacilli in sputum smears in pulmonary tuberculosis. Chest 1999; 116: 726-731.

31 Palomino JC. Nonconventional and new methods in the diagnosis of tuberculosis: feasibility and applicability in the field. Eur Respir J 2005; 26: 339-350.

32 Srisuwanvilai LO, Monkongdee P, Podewils LJ, et al. Performance of the BACTEC MGIT 960 compared with solid media for detection of Mycobacterium in Bangkok, Thailand. Diagn Microbiol Infect Dis 2008; 61: 402-407. 